\title{
Research on Plant Leaf Recognition Based on BP Neural Network
}

\author{
Xiaolei $\mathrm{Lu}^{1}$, Jing Qiu², \\ ${ }^{1}$ School of Engineering, Yunnan University of Business Management, Kunming 650106, Yunnan, China \\ ${ }^{2}$ Teaching Affairs Department, Yunnan Agricultural University, Kunming 650201, Yunnan, China
}

\begin{abstract}
Aiming at the problems of single plant identification feature, complex algorithm and unsatisfactory recognition rate, this research proposes to use BP algorithm to establish a plant leaf identification model. This research pre-processed the image of plant leaves, and extracted 14 feature variables of the image, including colour features, shape features and texture features. Using SPSS 21.0 software to perform principal component analysis on the extracted 14 feature variables, the analysis obtained 6 the main ingredient. Using the recognition model to carry out a simulation experiment, the experimental results show that when the number of hidden layers of the model is 1 , the number of hidden layer nodes is 6 , the accuracy of model recognition is better, and the average accuracy of model recognition is $95.56 \%$. From the analysis of model performance, the training accuracy and recognition accuracy of the model did not fluctuate greatly, and the graph changes relatively smoothly; when learning times is 400 times, the training and testing accuracy of the model reached more than $95 \%$. It shows that the model has high accuracy and strong generalization ability, which provides reference for plant leaf recognition research.
\end{abstract}

\section{Introduction}

Plants are the most important source of food and energy for human beings. Plants have genetic diversity, species diversity, ecological habits and ecosystem diversity. The shape and color of the same species are different, which brings great challenges to the effective identification of plants. Traditional plant classification and identification methods are generally artificial identification by botanists, which will inevitably have problems such as strong subjectivity, high professional ability, and low identification effect.

With the development of computer vision and digital image processing technology, the application of computer vision, digital image processing and other technologies to the classification and recognition of plants breaks through the limitations of traditional expression-based retrieval, avoids the subjectivity of manual description, and greatly reduces The workload has improved the accuracy and efficiency of plant classification.

Wu Qingfeng [1] studied content-based image retrieval of Chinese herbal medicine plants, extracting visual features in the fields of leaf shape, leaf veins, leaf teeth, etc. Experiments show that the retrieval accuracy of the application field features is high. Chen Xiaofen [2] used SVM classification method to achieve flower image retrieval, and the recognition rate of some flowers was as high as $95 \%$. Yan Yizhen [3] studied the use of leaf edge features for plant classification and retrieval. 30 nondivided leaf plants were divided into multiple leaf nodes, and the average global accuracy was better than $81.21 \%$. Yao Fei [4] divided 31 broad-leaved plants into 8 leaf nodes, with an average global classification accuracy of 94.19\%. Tan Liang [5] used AlexNet and GoogLeNet convolutional neural network to build a Chinese herbal medicine plant image model, and conducted experimental analysis, and its test results are good. Sun Jun [6] and others improved the traditional neural network and obtained 8 improved models, which realized the recognition of 26 types of diseases in 14 different plants, with a recognition rate of $99.56 \%$.Shijin Kumar P.S.[7] has been proposed a highly robust and efficient shape descriptor based CBIR model. Canny edge detection algorithm is employed to identify strong key points on the edges. Extracted features are fed to Support Vector Machine (SVM) classifier. The proposed model provides superior performance while comparing with existing retrieval models. He Jiean [8] proposed a method for identifying imperfect grains of wheat based on image detail enhancement, adding a BN layer to LeNet-5, ResNet-34, and VGG-16 respectively as a classification network. After testing, the improved classification networks are all the accuracy of the test set is improved by more than $7 \%$.

This research intends to use the deep neural network algorithm to build a plant leaf classification and recognition model, and conduct recognition experiments on six plant leaves of plant mint leaves, ginkgo leaves, mulberry leaves, maple leaves, apple leaves, and pear leaves to verify the effectiveness of the model. The

\footnotetext{
* Corresponding author: 2008004@ynau.edu.cn
} 
identification technology of plant leaves provides reference and reference.

\section{DATA AND MATERIALS}

\subsection{Data source}

The image data of this study, part of the image was taken by Canon $800 \mathrm{D}$ digital camera, and part of the image was downloaded from the Internet. To ensure that the images downloaded on the Internet are accurate and reliable, the python scripting language is used to delete duplicate images; images with a resolution less than 500 pixels are deleted. Finally, there are 3740 original images available, including six plant images of mint leaves, ginkgo leaves, mulberry leaves, maple leaves, apple leaves, and pear leaves. In order to reduce the over-fitting of the image set during the training phase, image enhancement technology is used to operate on the original image, each type of sample set is expanded to 1000 , and the total sample set is 6000 . Make labels for the sample set, and use a, $\cdots$, f to represent the corresponding category labels. An example sample is shown in Figure 1.
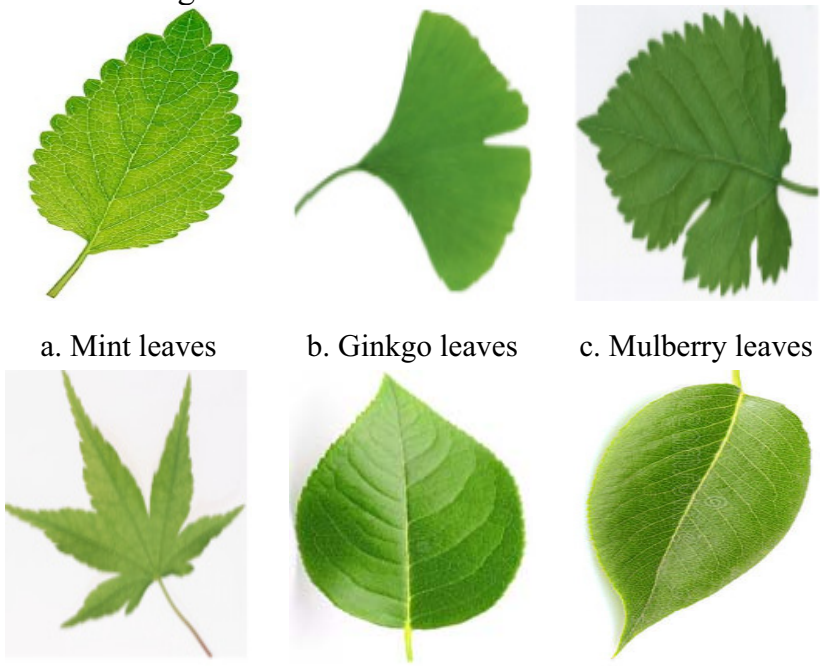

d. Maple leaves vision. There are three methods for color feature extraction, color histogram, color moment and color set. In this study, color moments are used to extract three components of the first-order moment (mean), secondorder moment (viarance), and third-order moment (skewness) of the image.

The evaluation criteria for shape feature extraction are: the zoom, translation and rotation of the shape do not affect the feature extraction; the descriptors of similar images are consistent and complete; a small amount of feature data can describe the shape features of the image; the descriptors should be universal. The Fourier descriptor is invariant to rotation, scaling and translation, and the shape feature is extracted using the Fourier descriptor. The complex coordinate function in the Fourier descriptor of the image is extracted, and Fourier transform is performed on it, and the first 7 modulus values in the frequency domain are normalized to obtain 6 shape features of the image.

The extraction of texture features mainly includes gray level co-occurrence matrix, local binary mode, and edge frequency and so on. In this study, the gray-level co-occurrence matrix was used to extract the average values of the five parameters of image entropy, energy, inverse gap, angular second moment, contrast, and correlation as the texture features of the image.

\subsection{Identification model construction}

BP neural network is a multi-layer forward network based on BP algorithm. It can solve linearly separable problems well, so it is widely used in prediction, classification, recognition and other related fields.

This research uses BP neural network to construct a plant leaf recognition model. Its network architecture consists of an input layer, a hidden layer and an output layer. The principle is to continuously correct the connection weights and thresholds through signal forward transmission and error reverse transmission to make the model the actual output value is constantly close to the expected output value, thereby improving the efficiency of model recognition.

1) Determination of the number of nodes in the input layer

In order to obtain effective image feature parameters, reduce the complexity of the model, and improve the effectiveness and efficiency of the model. This research first uses SPSS 21.0 to perform principal component analysis on the 14 colour features, shape features and texture features of the image to extract the effective feature parameters of the image. The analysis results are shown in Table 1.

Image feature extraction is the key to plant recognition and classification, mainly extracting the color, shape and texture features of the image.

Color feature extraction converts the pixel values in the image into numerical values. Because it does not require a lot of calculation and has low complexity, it is used as a better feature extraction method in computer

Table 1. Results of principal component analysis

\begin{tabular}{ccccccc}
\hline \multicolumn{4}{c}{ Explained total variance } \\
\hline \multirow{3}{*}{ Ingredients } & Total & Initial eigenvalue & \multicolumn{3}{c}{ Extract the sum of squares and load } \\
1 & 6.707 & 47.905 & 47.905 & 6.707 & 47.905 & 47.905 \\
\hline
\end{tabular}




\begin{tabular}{rcccccc}
\hline 2 & 2.008 & 14.343 & 62.248 & 2.008 & 14.343 & 62.248 \\
3 & 1.420 & 10.140 & 72.387 & 1.420 & 10.140 & 72.387 \\
4 & 1.335 & 9.536 & 81.923 & 1.335 & 9.536 & 81.923 \\
5 & .672 & 4.802 & 86.725 & .672 & 4.802 & 86.725 \\
6 & .652 & 4.656 & 91.381 & .652 & 4.656 & 91.381 \\
7 & .498 & 3.554 & 94.936 & & \\
8 & .252 & 1.801 & 96.737 & & \\
9 & .195 & 1.394 & 98.131 & & \\
10 & .122 & .869 & 99.000 & & \\
11 & .104 & .742 & 99.742 & & \\
12 & .024 & .172 & 99.914 & & \\
13 & .007 & .050 & 99.964 & & \\
14 & .005 & .036 & 100.000 & & \\
Extraction method: principal component analysis.
\end{tabular}

According to the analysis results in Table 1, when the component is 6 , its cumulative variance is $91.381 \%$, which can describe most of the image information. Therefore, the number of input nodes of the model is 6 .

2) Determination of the number of output nodes

The output node of this study is the type of plant leaf, which is a unique value, so the number of output nodes is 1 .

3) The determination of the number of hidden layer nodes and the number of hidden layers needs to be discussed through experiments to determine the optimal number of hidden layers and hidden layer nodes.

In summary, the input layer node of the plant leaf recognition model is 6 , and the output layer node is 1 . The recognition model is shown in Figure 2.

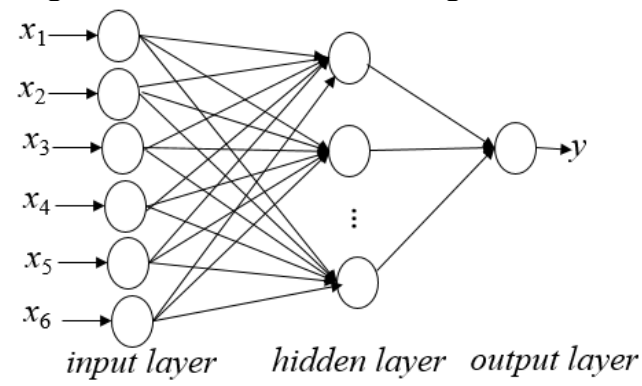

Fig. 2. Plant leaf recognition model

\section{Results and analysis}

In this study, the original sample set is divided into three image subsets: training set, validation set and test set. The training set accounts for $80 \%$, the validation set accounts for $10 \%$, and the test set accounts for $10 \%$, that is, the training set of each type of plant leaf image 800 frames, 100 frames in the verification set, and 100 frames in the test set. Each training set is used for feature extraction, model parameters are determined, the verification set is input to the determined recognition model for verification, and the model parameters are continuously adjusted until the optimal model is determined; the test set is input to the optimal model for recognition classification. Each experiment is performed
100 times, and the average of the experimental results is calculated.

\subsection{Determination of the number of hidden layers and the number of nodes}

In this experiment, the number of learning times, the learning rate, and the hidden function nodes of each layer are all 5. Each experiment is performed 100 times, and the average recognition accuracy and learning time are calculated to determine the number of hidden layers. The results are shown in Table 2 .

Table 2. The recognition accuracy and learning time of different hidden layer models

\begin{tabular}{cccccc}
\hline $\begin{array}{c}\text { Number of } \\
\text { Hidden layer }\end{array}$ & 1 & 2 & 3 & 4 & 5 \\
\hline $\begin{array}{c}\text { Learning times } \\
(\mathrm{s})\end{array}$ & 635 & 1025 & 1492 & 2054 & 2795 \\
Accuracy (\%) & 96.68 & 96.79 & 95.01 & 92.37 & 91.76 \\
\hline
\end{tabular}

It can be seen from Table 2 that when the number of hidden layers is 2 , the recognition accuracy is $96.79 \%$ and the learning time is $1025 \mathrm{~s}$; when the number of hidden layers is 1 , the recognition accuracy is $96.68 \%$, and the learning time is $635 \mathrm{~s}$, and its accuracy The accuracy is only $0.11 \%$ lower than when the number of hidden layers is 2, but the learning time is 390 s less. Comprehensive consideration, the number of hidden layers of the recognition model is determined to be 1 , that is, the model adopts a three-layer network architecture.

This experiment studies the effect of the number of hidden layer nodes on the recognition accuracy and training time. The number of hidden layers is 2 layers. The number of neurons, the number of learning times, and the learning rate of the hidden layer in each experiment are the same, with different hidden layers. Perform 100 experiments with the number of nodes in the hidden layer to find the average recognition accuracy and learning time to determine the number of nodes in the hidden layer. The results are shown in Table 3. 
Table 3. The recognition accuracy and learning time of different hidden layer nodes

\begin{tabular}{ccccccccc}
\hline Number of hidden layer nodes & 3 & 4 & 5 & 6 & 7 & 8 & 9 & 10 \\
\hline Learning times (s) & 682 & 759 & 986 & 1078 & 1438 & 1763 & 1859 & 2056 \\
Accuracy (\%) & 88.63 & 90.92 & 94.65 & 96.83 & 96.89 & 94.14 & 92.51 & 89.74 \\
\hline
\end{tabular}

It can be seen from Table 3 that as the hidden layer nodes increase, the learning time of the model is longer. This is because the increase of hidden layer nodes increases the complexity of the model and the learning time will be longer. When the hidden layer node is 6 , the recognition accuracy rate is $96.83 \%$, and the learning time is $1078 \mathrm{~s}$; when the hidden layer node is 7 , the recognition accuracy rate is $96.89 \%$, and the learning time is $1438 \mathrm{~s}$. Although when the hidden layer node is 7 , the recognition accuracy is slightly higher than that of the hidden layer node 6 , but the time has increased by nearly 400s. Considering the two factors of time and accuracy, the number of hidden layer nodes in this study is taken as 6 .

\subsection{Model performance analysis}

The model is used to classify and identify six plant leaves, and the accuracy of the training set and test set is simulated and analyzed to analyze the convergence performance of the model. The simulation analysis result is shown in Figure 3.

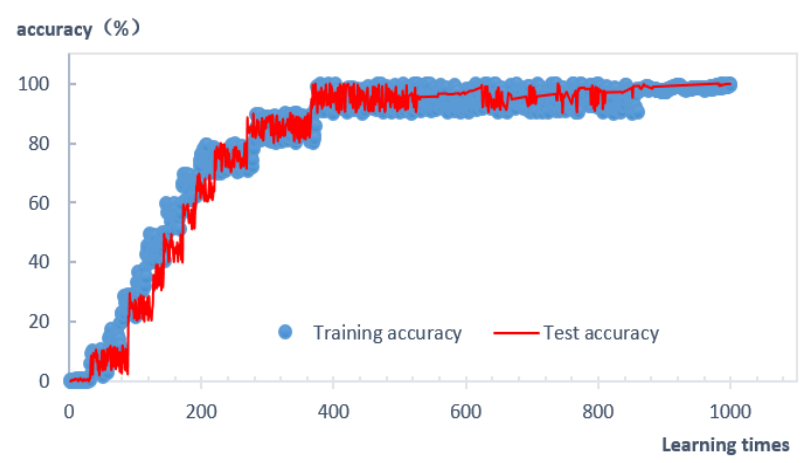

Fig. 3. Analysis of model training accuracy and test accuracy

According to the analysis results in Figure 3, the model recognition accuracy rate increases with the increase of the number of learning, the training accuracy rate and the test accuracy rate continue to increase, the image does not show large fluctuations. When learning times is 400 times, the graphics of training accuracy and test accuracy tend to be stable, and the accuracy of training and testing is above $95 \%$. It shows that the model has strong generalization ability and good convergence.

\subsection{Model validity analysis}

In this experiment, the model was used to identify and verify the leaf images of 6 plants of ginkgo leaves, mint leaves, maple leaves, mulberry leaves, apple leaves and pear leaves. The experimental results are shown in Table 4.
Table 4. Plant leaf recognition result

Plant leafGinkgo Mint Maple Mulberry Apple Pear Average

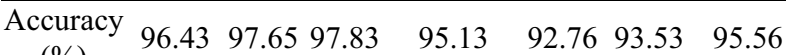

It can be seen from Table 4 that the model has the highest recognition accuracy of maple leaves, which is $97.83 \%$. The recognition accuracy of each leaf is above $90 \%$, and the average recognition accuracy is $95.56 \%$. That the model is effective.

\section{Conclusion}

This study first preprocessed the image of plant leaves, extracted 14 colour, shape and texture features, and performed principal component analysis on 14 factors using SPSS 21.0, and extracted 6 factors. The BP algorithm is used to establish a plant leaf recognition model, and the following conclusions are obtained:

1) The study found that when the number of hidden layers is 1 , the number of hidden layers node is 6 , the recognition accuracy of the model is better.

2) The simulation analysis of the model performance showed that the training accuracy and recognition accuracy graphs did not show large fluctuations. After 400 trainings, the training and testing accuracy reached more than $95 \%$, indicating that the model is stable.

3) The model is used to study the identification of 6 plant leaves. From the experimental results, the average identification accuracy rate is $95.56 \%$, indicating that the method is effective and feasible.

The study found that plant leaves with similar characteristics are easily misidentified, which reduces the recognition effect of the model. Therefore, how to collect a large number of high-resolution samples, obtain typical plant characteristics, and improve the recognition effect of plant leaves is an urgent problem to be solved.

\section{Acknowledgments}

This research was supported by the Scientific Research Fund Project of Yunnan Provincial Department of Education "Research on Intelligent Recognition of Rice Disease Image Based on Computer Vision and BP Network" (Project Number: 2016ZZX103), and Yunnan Science and Technology Major Project"R\&D and application demonstration for key technologies of digital agriculture of Yunnan plateau characteristics" (Project Number: 202002AE090010). 


\section{References}

1. Wu Qingfeng. (2007)Research on key techniques of content-based Chinese herbal medicine botanic image retrieval. Xiamen University.

2. Chen xiaofen. (2009)Content-Based flower image retrieval. Xiamen University.

3. Yan Yi-zhen, Zhou Jianhua. (2015)Plant image classification and retrieval based on leaf margin features. Journal of East China Normal University ( Natural Science), (04):154-163.

4. Yao Fei, Ye Kang, Zhou Jianhua. (2015)Automatic image classification and retrieval by analyzing plant leaf features. Journal of Zhejiang A \& F University, 32(03):426-433.

5. Liang Tan. (2016)Key technology research of content-based Chinese herbal medicine image search. Zhejiang University.

6. Sun Jun, Tan Wenjun, Mao Hanping, Wu Xiaohong, Chen Yong, Wang Long. (2017)Recognition of multiple plant leaf diseases based on improved convolutional neural network. Transactions of the Chinese Society of Agricultural Engineering (Transactions of the CSAE), 33(19): 209 - 215.

7. Shijin Kumar P.S.,Udaya Kumar Naluguru,Ushasree A.,Sumalata G.L.. (2020)Key point oriented shape features and SVM classifier for content based image retrieval. Materials Today: Proceedings, (prepublish).

8. He Jiean, Wu Xiaohong, He Xiaohai, Hu Jianrong, Qing Linbo. (2021)Imperfect wheat kernel recognition combined with image enhancement and conventional neural network. Journal of Computer Applications: 1-7[2021-01-28]. 